\title{
Properties of Mixing Length and Dispersive Stress in Airflows over Urban-Like Roughness Obstacles with Variable Height
}

\author{
Toshiya Yoshida and Tetsuya Takemi \\ Disaster Prevention Research Institute, Kyoto University, Uji, Kyoto, Japan
}

\begin{abstract}
Effects of obstacle-height variability on mixing length and dispersive stress are investigated by conducting large-eddy simulations of airflows over arrays of roughness obstacles with variable height. We evaluate differences among three simulations of flows over obstacles with no, moderate, and high obstacle-height variability. Within the canopies, effective mixing length shows one local maximum and minimum in the simulation with no obstacleheight variability but two maxima and minima in the simulations with obstacle-height variability. The number of the local maxima and minima corresponds to that of the shear layers seen at the heights of obstacle tops. Enhanced dispersive stress appears within the canopy between the heights of the lower- and higher-obstacle tops in the simulations with obstacle-height variability. Particularly in the simulations with high obstacle-height variability, the magnitude of dispersive stress becomes comparable to that of the Reynolds stress at the height of the lower-obstacle top. These results suggest that actual urban areas with high building-height variability should significantly affect properties of mixing length and dispersive stress.
\end{abstract}

(Citation: Yoshida, T., and T. Takemi, 2018: Properties of mixing length and dispersive stress in airflows over urban-like roughness obstacles with variable height. SOLA, 14, 174-178, doi:10.2151/sola.2018-031.)

\section{Introduction}

Urban effects on the atmosphere appear as changes in air quality and the thermal and wind environment. Such effects are incorporated in numerical weather prediction models as an urban canopy parameterization (UCP). UCPs generally take into account two effects: a dynamical effect that includes drag by buildings and resulting momentum transport; a thermal effect due to sensible heat flux from urban surfaces (Martilli et al. 2002; Santiago and Martilli 2010). If the horizontal heterogeneity of urban surfaces becomes high, representing the urban effects in UCP becomes complicated. This study focuses on the influences of the heterogeneity of urban surfaces on the properties of vertical momentum transport.

Generally, the momentum flux over heterogeneous surfaces is composed of the Reynolds and dispersive stresses (Raupach and Shaw 1982). Reynolds stress can be parameterized with mixing length $l_{m}$. The forms of $l_{m}$ with urban effects were developed by Coceal and Belcher (2004) (referred to as CB04), Kondo et al. (2015), and others. In their studies, $l_{m}$ increases monotonically with height within the urban canopy as well as above the canopy top. In contrast, Castro (2017) showed that $l_{m}$ obtained from direct numerical simulation (DNS) and large-eddy simulation (LES) of flows over roughness obstacles with uniform height has a single local maximum within the canopy, and that $l_{m}$ has multiple local maxima within the canopy for flows over roughness with variable height. In this way, there are still unknowns on how and why the characteristics of $l_{m}$ appear depending on the urban geometrical feature.

Corresponding author: Toshiya Yoshida, Disaster Prevention Research Institute, Kyoto University. Kyoto University, Gokasho, Uji, Kyoto 6110011, Japan. E-mail: yoshida_t@storm.dpri.kyoto-u.ac.jp.
Furthermore, dispersive stress was shown to have contributions equal to or greater than that of Reynolds stress to the total momentum flux within an urban canopy (Coceal et al. 2006). Meanwhile, Castro (2017) indicated that the mixing length determined by the sum of Reynolds and dispersive stresses does not differ significantly from that determined only by Reynolds stress. However, the maximum value of the former was shown to be up to approximately $20 \%$ greater than that of the latter, depending on the density and arrangement of obstacles. These studies examined the flows over roughness obstacles with uniform height. In contrast, in flows over roughness obstacles with variable height, dispersive stress extends to a higher altitude than in the case with uniform height (Xie et al. 2008; Yoshida et al. 2018). However, the relationship between the obstacle-height variability and dispersive stress has not been sufficiently investigated.

This study investigates the properties of mixing length and dispersive stress for flows over roughness obstacles with variable height by conducting LES of turbulent flows.

\section{Numerical experiment and analysis procedure}

\subsection{Model and experimental design}

This study uses the LES model used and described in Yoshida et al. (2018). To represent the effects of obstacles on flows, the model employs the feedback forcing by Goldstein et al. (1993) (see Supplement 1). The size of the main computational domain is $4 \mathrm{~km} \times 2.4 \mathrm{~km} \times 1.026 \mathrm{~km}$ in the streamwise, spanwise, and vertical directions (Fig. 1a). The horizontal grid spacing is $2 \mathrm{~m}$, while the vertical grid spacing is $2 \mathrm{~m}$ below $350 \mathrm{~m}$ and stretched with height up to $16 \mathrm{~m}$.

In addition to the main computational domain, we set a driver region to accelerate the generation of turbulent flows which are imposed at the inlet boundary of the main domain. At the outlet boundary of the main domain, the Sommerfeld radiation condition is imposed: the flows at the boundary are advected with the representative streamwise velocity at the outlet plane. A periodic condition is imposed at the lateral boundaries. The top boundary condition is free-slip and zero-speed for the horizontal and vertical velocity components, respectively. Monin-Obukhov similarity theory is applied at the bottom boundary not masked by obstacles.

We conducted three simulations of turbulent flows over square

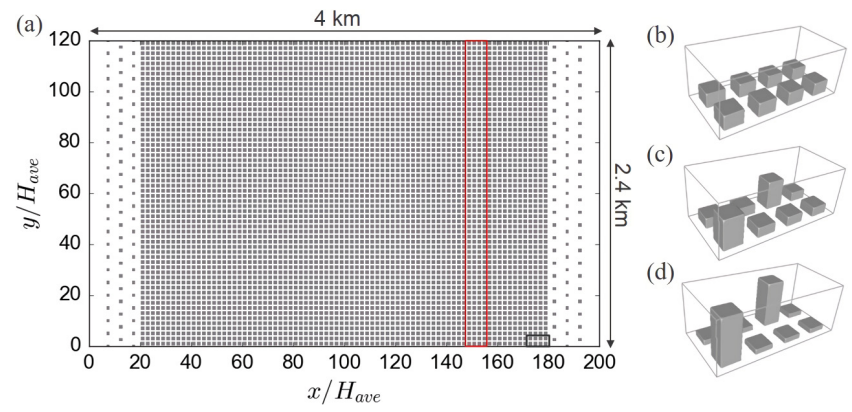

Fig. 1. (a) Plan view of the computational domain and obstacle layouts in the unit area of (b) V00, (c) V05, and (d) V10. The black and red rectangle indicates a unit area and region conducting spatial average, respectively. 
arrays of obstacles with different obstacle-height variability $V_{h}$ (the ratio of the standard deviation of obstacle height to the average obstacle height $H_{\text {ave }}$ ). These simulations have the same frontal area index $\lambda_{f}$ (the ratio of the frontal area of buildings to the total surface area) and $H_{\text {ave }}$, which are set to 0.25 and $20 \mathrm{~m}$, respectively. We examine cases with no variability $\left(V_{h}=0.0\right.$, referred to as V00), moderate variability $\left(V_{h}=0.5\right.$, V05), and high variability $\left(V_{h}=1.0, \mathrm{~V} 10\right)$. Unit areas in each simulation are shown in Figs. $1 \mathrm{~b}, 1 \mathrm{c}$, and 1d. The computational domains consist of $20 \times 30$ unit areas in the streamwise and spanwise directions, with buffer areas around the inlet and outlet boundaries to stabilize the computations (Fig. 1a). Each unit area consists of eight cubes with the height of $H_{\text {ave }}$ in V00 or two higher (with the height of $H_{\max }$ ) and six lower (with the height of $H_{\min }$ ) rectangular obstacles in V05 and V10 (see Table 1 for $H_{\max }$ and $H_{\min }$ ). The obstacles in the three experiments have the same length and width of $H_{\text {ave }}$. The horizontal area of each obstacle is resolved with 10 by 10 grid points, while vertical grid points $n_{z}$ depends on the obstacles (see Table 1). For the analysis, the spatial average is conducted in the region spanning from 2960 to $3120 \mathrm{~m}$ in the streamwise direction (shown as the red rectangle in Fig. 1a). This region is chosen because space-averaged vertical velocity is nearly zero. The integration time for all the simulations is 5,400 s. The time is equal to approximately $8 H_{x} / U_{\infty}$, where $U_{\infty}$ and $H_{x}$ are freestream velocity and the streamwise domain size. The last 1,800 s $\left(\sim 2.7 H_{x} / U_{\infty}\right)$ is used for the analysis.

\subsection{Analysis procedure for mixing length}

In $\mathrm{CB} 04$ it was considered that the mixing length is proportional to the distance from the surface within a sparse canopy but is constant within a dense canopy. By combining the characteristics of both canopies, CB04 represented the mixing length, denoted here as $l_{m, C B}$, as follows:

$$
\frac{1}{l_{m, C B}}=\frac{1}{\kappa z}+\frac{1}{l_{c}} \text { (below the canopy height) }
$$

and

$$
l_{m, C B}=\kappa(z-d) \text { (above the canopy height), }
$$

Table 1. Surface characteristics for each simulation. $H_{\text {ave }}, H_{\max }, H_{\min }$, and $n_{z}$ are average obstacle height, higher obstacle height, lower obstacle height, and vertical grid points, respectively.

\begin{tabular}{ccccccc}
\hline Case & $V_{h}$ & $H_{\max } / H_{\text {ave }}$ & $H_{\min } / H_{\text {ave }}$ & $n_{z}\left(H_{\max }\right)$ & $n_{z}\left(H_{\min }\right)$ & $d / H_{\text {ave }}$ \\
\hline V00 & 0.0 & 1.00 & 1.00 & 10 & 10 & 0.823 \\
V05 & 0.5 & 1.95 & 0.68 & 19 & 7 & 1.410 \\
V10 & 1.0 & 2.90 & 0.37 & 29 & 4 & 2.030 \\
\hline
\end{tabular}

where $\kappa$ is the von Kármán constant, $z$ is height, $d$ is the displacement height, and $l_{c}$ is constant mixing length. Here the canopy height corresponds to $H_{\text {ave }}\left(=H_{\max }\right)$ in $\mathrm{V} 00$ and $H_{\max }$ in $\mathrm{V} 05$ and V10. $l_{c}$ can be derived from the Eq. (1) and (2) at the canopy height. The displacement height is obtained from log-law fitting of the streamwise velocities. Table 1 shows these values in each simulation. Note that $d / H_{\text {ave }}$ in V00 is similar to that obtained from the wind-tunnel experiment on flows over an aligned array of uniform-height obstacles in Cheng et al. (2007).

Coceal et al. (2006) defined effective mixing length calculated from the Reynolds stress $\left\langle\overline{u^{\prime} w^{\prime}}\right\rangle$ and vertical shear of streamwise velocity $\partial\langle\bar{u}\rangle / \partial z$ as

$$
l_{m, e f f}=\frac{\sqrt{-\left\langle\overline{u^{\prime} w^{\prime}}\right\rangle}}{\partial\langle\bar{u}\rangle / \partial z},
$$

where the overbar, brackets, and prime indicate temporal average, spatial average, and temporal deviation, respectively. We regard this effective mixing length as a baseline to evaluate the mixing-length properties.

We further examine a mixing length calculated from the sum of Reynolds and dispersive stresses to estimate the impacts of dispersive stress (Castro 2017). This mixing length $l_{m, \text { sum }}$ is determined as:

$$
l_{m, \text { sum }}=\frac{\sqrt{-\left\langle\overline{u^{\prime} w^{\prime}}\right\rangle-\left\langle\bar{u}^{\prime \prime} \bar{w}^{\prime \prime}\right\rangle}}{\partial\langle\bar{u}\rangle / \partial z},
$$

where $\left\langle\bar{u}^{\prime \prime} \bar{w}^{\prime \prime}\right\rangle$ is dispersive stress and double prime means spatial variation from the time-averaged value.

In the next section, we investigate the effects of obstacle-height variability on these three mixing lengths and how $l_{m, \text { eff }}$ is different from $l_{m, C B}$ and $l_{m, \text { sum }}$.

\section{Results}

Figures $2 \mathrm{a}$ and $2 \mathrm{~b}$ show the vertical profiles of time- and spaceaveraged streamwise velocity $\langle\bar{u}\rangle$ and Reynolds stress $\left\langle\overline{u^{\prime} w^{\prime}}\right\rangle$ normalized by $U_{\infty}$. The velocities above $H_{\text {ave }}$ decrease with the increase in $V_{h}$ because of the influences from higher obstacles. The maxima of Reynolds stress are seen at the canopy height and become larger with the increase in $V_{h}$. These features of the streamwise velocity and Reynolds stress are consistent with those in Nakayama et al. (2011). Figure 2c shows the vertical shears of the mean streamwise velocity normalized by $U_{\infty}$ and $H_{\text {ave }}$. The V00 case features a strong shear layer at the top of the canopy, whereas the pronounced shear layers are seen at the higher- and lower-obstacle heights in V05 and V10.

Figure 3 compares $l_{m, C B}$ and $l_{m \text {, eff }}$ in each simulation. For (a)

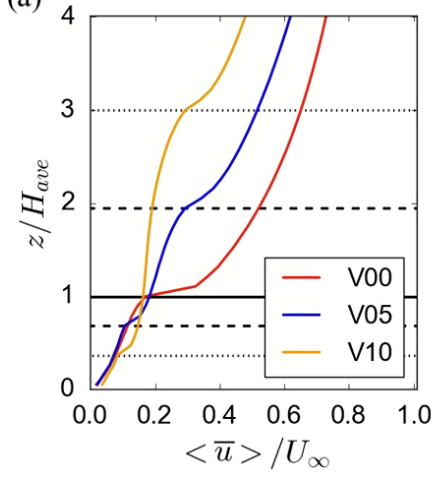

(b)

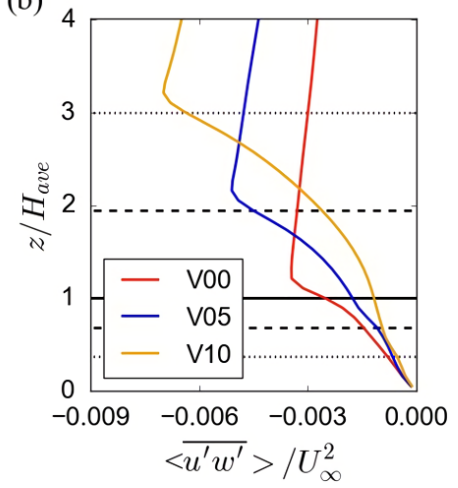

(c)

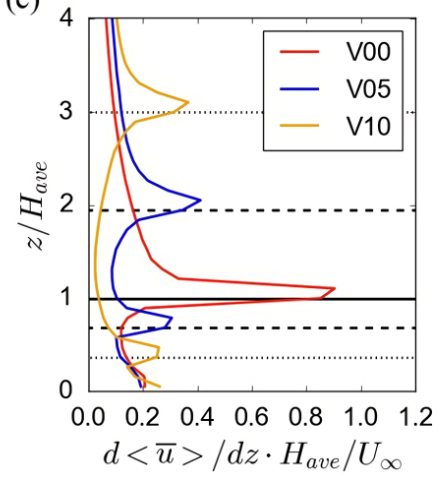

Fig. 2. Vertical profiles of temporal and spatial averaged (a) streamwise velocity, (b) Reynolds stress, (c) vertical shear of streamwise velocity. The black-solid, -dashed and -dotted lines indicate the height of obstacles in V00, V05, and V10, respectively. 
(a)

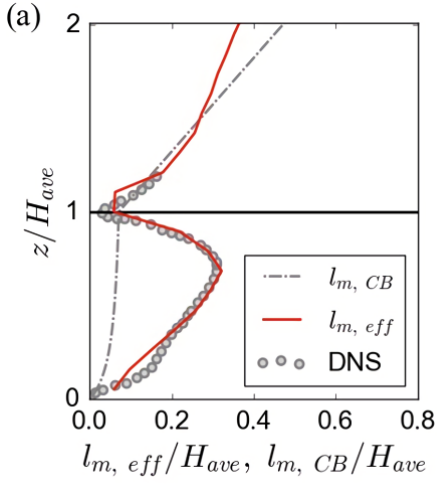

(b)

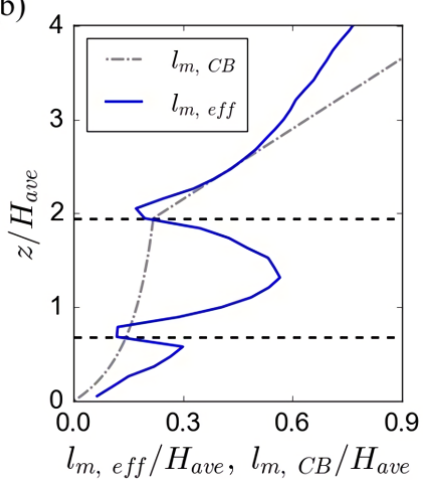

(c)

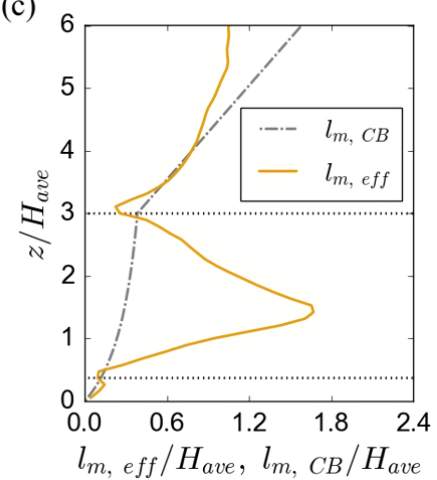

Fig. 3. Vertical profiles of mixing lengths obtained with Eq. (3) and the estimations of CB04 in (a) V00, (b) V05, and (c) V10. The gray circle denotes the profile obtained from the DNS result (Branford et al. 2011). The horizontal lines are the same as in Fig. 2.

(a)

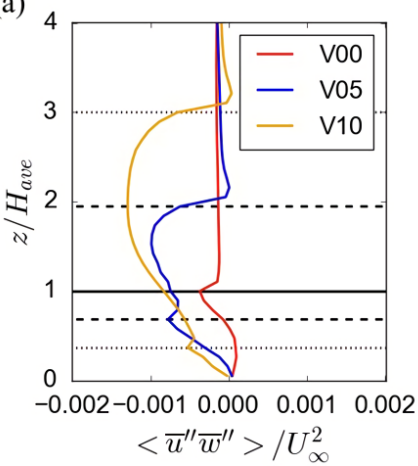

(b)

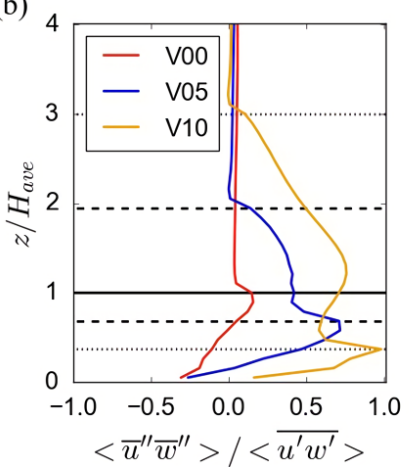

(c)

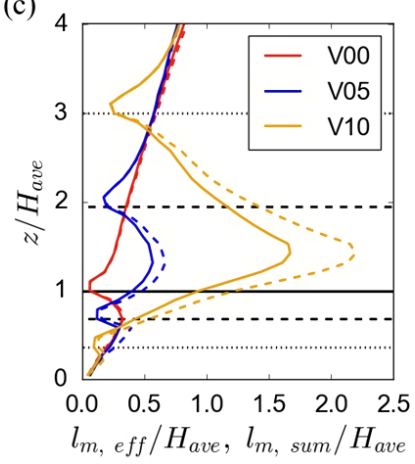

Fig. 4. Vertical profiles of (a) dispersive stress, (b) the ratio of dispersive stress to Reynolds stress, and (c) $l_{m, \text { eff }}$ (solid lines) and $l_{m, \text { sum }}$ (dashed lines). The horizontal lines are the same as in Fig. 2.

validating the present LES, the profile of $l_{m, \text { eff }}$ in V00 is compared with the DNS result of Branford et al. (2011) and Castro (2017), and seems to correspond well with the DNS result. Above the canopy, the profiles of $l_{m, C B}$ in all the simulations agree with those of $l_{m, \text { eff }}$ at and around the canopy top, while $l_{m, C B}$ gradually deviates from $l_{m, e f f}$ as the height increases. Within the canopies, $l_{m, C B}$ is significantly different from $l_{m, \text { eff }}$. The profile of $l_{m, \text { eff }}$ in V00 shows a local minimum at $H_{\text {ave }}$ because the strong shear layer near the canopy top (see Fig. 2c) restricts penetration of large eddies from above the canopy (Coceal et al. 2006; Kono et al. 2010). There is a local maximum at about $0.7 H_{\text {ave }}$, where the vertical shear indicates a local minimum (Fig. 2c). In contrast, $l_{m, \text { eff }}$ in V05 and V10 has two local minima and maxima below the canopy heights. The minima appear at the heights of two shear layers as shown in Fig. $2 \mathrm{c}$, while the maxima are located approximately at the heights of local minima of the vertical shear; in other words, the number of the local minima and maxima within the canopies corresponds to that of the shear layers seen at the heights of obstacle tops. In this way, $l_{m, \text { eff }}$ within the canopy of obstacles with variable height has multiple local maxima and minima as found in Castro (2017).

Figure 4 a shows dispersive stress $\left\langle\bar{u}^{\prime \prime} \bar{w}^{\prime \prime}\right\rangle$ normalized by $U_{\infty}$. The sign changes at the middle of the canopy in V00. This feature is consistent with that found in flows over roughness obstacles with uniform height (Coceal et al. 2006). In contrast, the dispersive stresses in V05 and V10 are mostly negative within the canopy. Furthermore, in V05 and V10 the peaks of the dispersive stress appear in the upper part of the canopy. In Fig. 4b, the ratios of the dispersive stress to the Reynolds stress are found to increase within the canopy with the increase in $V_{h}$. In particular, the ratio is greater than 0.5 at around the height of the lower-obstacle top in $\mathrm{V} 05$, and becomes nearly 1 at the lower-obstacle top in V10.

The features indicated in Figs. $4 \mathrm{a}$ and $4 \mathrm{~b}$ determine the profiles of the mixing lengths. Figure 4c demonstrates that in all the simulations the shapes of $l_{m \text {, sum }}$ are similar to those of $l_{m \text {, eff }}$. Quantitatively, the maximum values of $l_{m, \text { sum }}$ in V00, V05, and V10 are respectively $2 \%, 18 \%$, and $31 \%$ larger than those of $l_{m \text {, eff }}$. This 2-\% difference in V00 is much smaller than that found in Castro (2017) who showed that the maximum value of $l_{m, \text { sum }}$ are about $12 \%$ larger than that of $l_{m \text {,eff }}$ over aligned-type arrays of uniform-height obstacles. As shown in Fig. 3a, $l_{m \text {, eff }}$ calculated only from Reynolds stress is consistent with that in the DNS result. Therefore, we consider that the dispersive stress in V00 is underestimated because of an insufficient resolution to represent each roughness obstacle.

To examine distributions of dispersive stress fraction $\bar{u}^{\prime \prime} \bar{w}^{\prime \prime}$, Figs. 5 and 6 show the horizontal and vertical cross-sections of $\bar{u}^{\prime \prime} \bar{w}^{\prime \prime}$ and the flow fluctuations from the time-averaged flows in $\mathrm{V} 00$ and V10. We found that the profiles of V05 were qualitatively similar to those of V10, and thus we evaluate differences in the distributions between V00 and V10. The distributions are obtained from ensemble averages for the 30 unit areas within the red rectangle in Fig. 1a. The streamwise and spanwise coordinates in Figs. 5 and 6 are represented as local coordinates $\left(x^{\prime}\right.$ and $\left.y^{\prime}\right)$. Note that those time-averaged spanwise and vertical velocities $\bar{v}$ and $\bar{w}$ were almost the same as the spatial deviations. Thus, $\bar{v} \sim \bar{v}^{\prime \prime}$ and $\bar{w}$ $\sim \bar{w}^{\prime \prime}$.

Figure 5a shows that positive and negative $\bar{u}^{\prime \prime} \bar{w}^{\prime \prime}$ is concentrated in the front and back sides of the obstacles, respectively, in the $x-y$ plane of V00. This result is similar to the distributions in similar obstacle arrays (Coceal et al. 2007; Martilli and Santiago 2007). The vectors in Fig. 5a indicates that $\bar{u}^{\prime \prime}$ is overall negative between the obstacles. Thus, the sign of $\bar{u}^{\prime \prime} \bar{w}^{\prime \prime}$ depends on that of $\bar{w}^{\prime \prime}$ (Rasheed and Robinson 2013). Figure 5b shows that negative and positive $\bar{w}^{\prime \prime}$ exists in the front and back sides of the obstacles, 


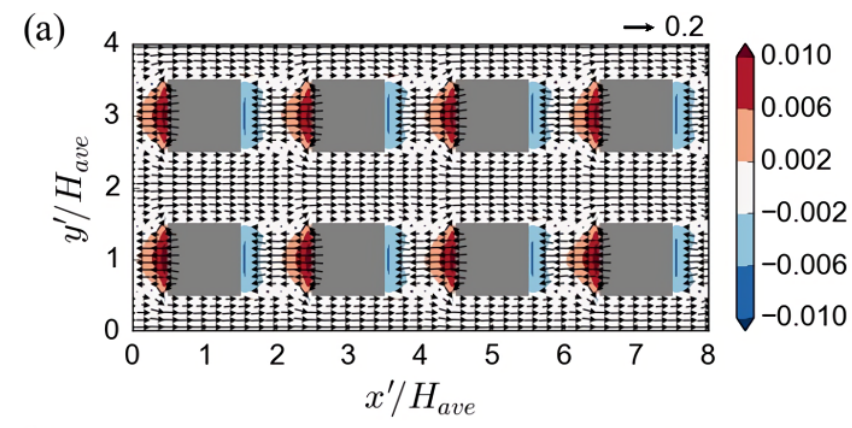

(b)
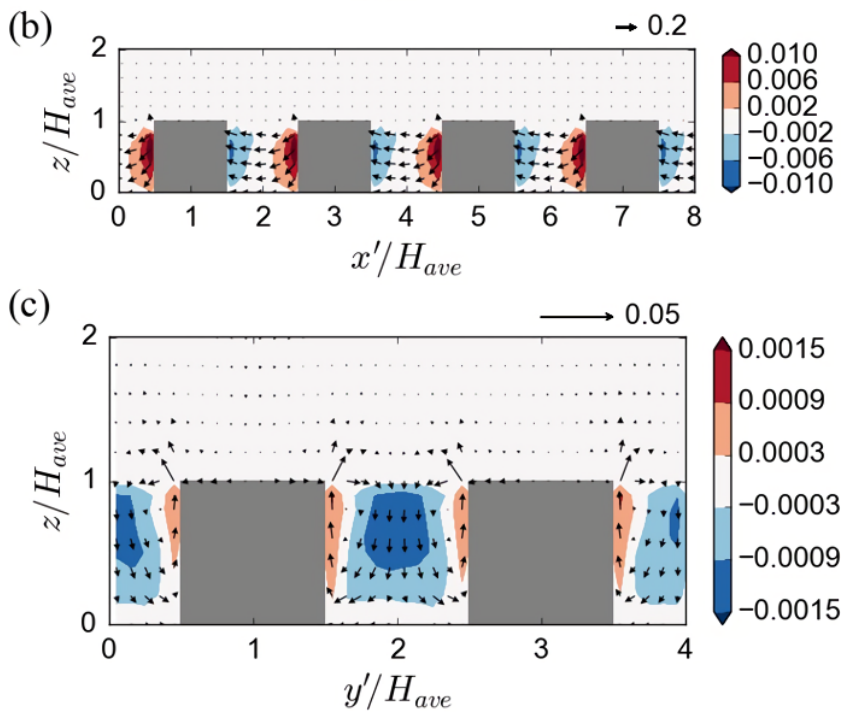

Fig. 5. Distributions of $\bar{u}^{\prime \prime} \bar{w}^{\prime \prime}$ (shaded) in (a) x-y slice at $z=0.5 H_{\text {ave }}$, (b) $\mathrm{x}-\mathrm{z}$ slice at $y^{\prime}=3 H_{\text {ave }}$, and (c) $\mathrm{y}-\mathrm{z}$ slice at $x^{\prime}=5 H_{\text {ave }}$ in V00. Each figure includes the vectors of $\bar{u}^{\prime \prime}$ and $\bar{v}^{\prime \prime}, \bar{u}^{\prime \prime}$ and $\bar{w}^{\prime \prime}$, and $\bar{v}^{\prime \prime}$ and $\bar{w}^{\prime \prime}$, respectively.

respectively, because cavity vortices between the obstacles cause downdraft at the front side and updraft at the back side. Therefore, positive and negative $\bar{u}^{\prime \prime} \bar{w}^{\prime \prime}$ appear in the front and back regions, respectively. In contrast, negative $\bar{u}^{\prime \prime} \bar{w}^{\prime \prime}$ is dominant along the streamwise streets (Fig. 5c), although the magnitude is much smaller than that between the obstacles as seen in Fig. 5a.

Figure 6a shows that, in the case of V10, positive $\bar{u}^{\prime \prime} \bar{w}^{\prime \prime}$ is limited in the front side of the higher obstacles but negative $\bar{u}^{\prime \prime} \bar{w}^{\prime \prime}$ appears behind the higher obstacles and near the edge of the front side. This feature is similar to that in staggered-type arrays of obstacles with uniform height (Coceal et al. 2007). As demonstrated in Fig. 6 b, the positive and negative $\bar{u}^{\prime \prime} \bar{w}^{\prime \prime}$ are distributed in the front and back of the higher obstacle from the ground to the top. The vector fields indicate that the positive and negative $\bar{u}^{\prime \prime} \bar{w}^{\prime \prime}$ are composed of downdraft with low streamwise velocity $\left(\bar{u}^{\prime \prime}<0\right.$, $\left.\bar{w}^{\prime \prime}<0\right)$ and updraft with low streamwise velocity $\left(\bar{u}^{\prime \prime}<0, \bar{w}^{\prime \prime}\right.$ $>0$ ), respectively. The spanwise cross section (Fig. 6c) indicates that negative $\bar{u}^{\prime \prime} \bar{w}^{\prime \prime}$ exists around the side of the higher obstacles from the surface to about $z=2 H_{\text {ave }}$. From Figs. 6a and 6c, this negative $\bar{u}^{\prime \prime} \bar{w}^{\prime \prime}$ is caused by downdraft with high streamwise velocity $\left(\bar{u}^{\prime \prime}>0, \bar{w}^{\prime \prime}<0\right)$. These results indicate that the higher obstacles have a significant contribution to the production of dispersive stress within the canopy.

From the results in Figs. 4a and 4b, we consider that the influences of lower obstacles should not be ignored within the canopy. According to Fig. 6 a, the negative $\bar{u}^{\prime \prime} \bar{w}^{\prime \prime}$ around the areas between higher and lower obstacles in the spanwise direction (e.g. around the point $\left.\left(x^{\prime}, y^{\prime}\right)=\left(H_{\text {ave }}, 2 H_{\text {ave }}\right)\right)$ is considered to be enhanced due to the convergence of the streamwise velocities along the streamwise streets. There are other locations of the negative $\bar{u}^{\prime \prime} \bar{w}^{\prime \prime}$, which is found around the areas between higher and lower obstacles in the streamwise direction (e.g. around the point $\left(x^{\prime}, y^{\prime}\right)=\left(3.5 H_{\text {ave }}\right.$,
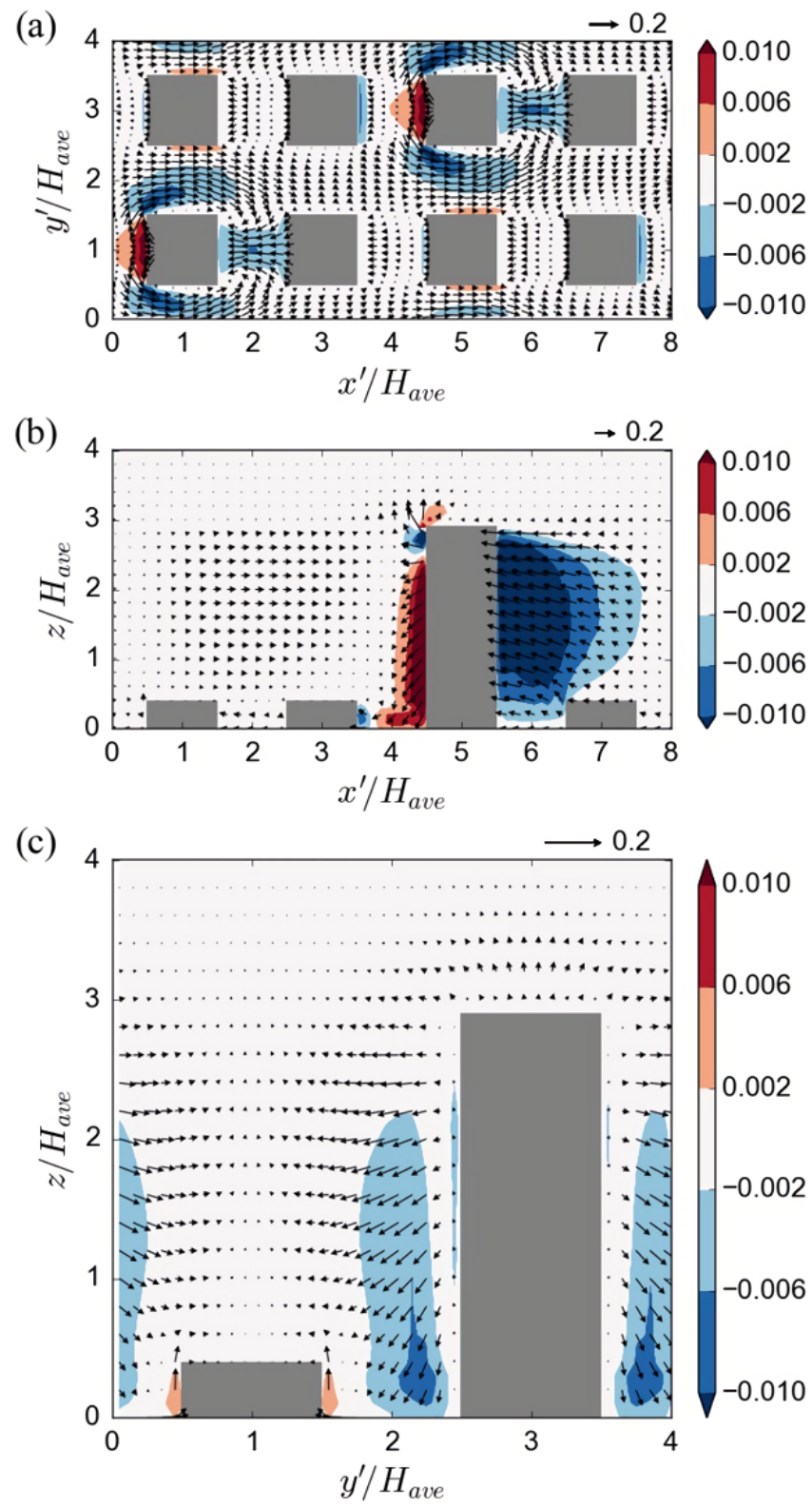

Fig. 6. As Fig. 5, except with the corresponding distributions of V10 and the $\mathrm{x}$-y slice at $z=0.37 H_{\text {ave }}$ (which is equal to the top of the lower obstacles).

$\left.3 H_{\text {ave }}\right)$ ), generated by strike of reversed flows from the front side of the higher obstacles toward the back side of the lower obstacles. Therefore, we consider that not only higher but also lower obstacles affect the distributions of $\bar{u}^{\prime \prime} \bar{w}^{\prime \prime}$ at heights within the canopy.

\section{Conclusions and discussion}

To investigate effects of obstacle-height variability on mixing length and dispersive stress, we conducted LESs of airflows over arrays of roughness obstacles with variable height and compared the results from three simulations in which obstacle heights had no, moderate, and high variability, respectively. The mixing lengths defined in Coceal and Belcher (2004) showed good agreement with the effective mixing lengths calculated from Reynolds stresses and vertical shears of streamwise velocity above the canopy heights regardless of obstacle-height variability. Within 
the canopies, the effective mixing lengths showed one local maximum and minimum in the no-variability simulation and two maxima and minima in the simulations with obstacle-height variability, although the mixing lengths of Coceal and Belcher in each simulation monotonically increased with the height. The number of the local maxima and minima was consistent with that of the shear layers seen at the heights of obstacle tops.

Dispersive stresses and their ratios to the Reynolds stresses tended to increase with obstacle-height variability. The magnitude of dispersive stress in the simulation with high obstacle-height variability was comparable to that of Reynolds stress at the lower-obstacle heights. Thus, the lower obstacles largely affected the production of the dispersive stresses at lower heights. The mixing lengths obtained from the sum of Reynolds and dispersive stresses were larger than those from Reynolds stress only and the differences became larger with obstacle-height variability.

Large values of dispersive stress fractions in the no-variability simulation were concentrated in the areas between the front and back sides of obstacles. In the simulations with obstacle-height variability, however, large values existed around the higher obstacles from the surface to the top. Therefore, the dispersive stresses within the canopies were generally affected by the higher obstacles. In contrast, around the lower-obstacle heights, it is considered that both the lower and higher obstacles affected the distributions of dispersive stress between the two-type obstacles.

In the simulations, the dispersive stress was underestimated from that obtained by DNS, owing to the insufficient resolution to represent each obstacle. Nevertheless, the characteristics of dispersive stress were qualitatively similar to the results of DNS. By increasing the resolution, it is expected that the contribution from dispersive stress becomes more significant.

This study demonstrated that the profiles of mixing length are more complicated over obstacles with obstacle-height variability than those with uniform height. Additionally, contribution of dispersive stress to momentum transport increases with obstacleheight variability. These results suggest that the effects of obstacleheight variability on the mixing length and dispersive stress should be enhanced over actual urban areas because of the obstacles with varying height.

\section{Acknowledgments}

The comments by anonymous reviewers are greatly acknowledged to improve the manuscript. This research partly used computational resources under the Collaborative Research Program for Young Scientists provided by the Academic Centre for Computing and Media Studies, Kyoto University. This study was supported by JSPS Kakenhi 16H01846 and 18H01680, and DPRI Collaborative Research 28H-04 and 29S-01.

Edited by: S.-H. Chen

\section{Supplements}

Supplement 1 shows details of the numerical model and the representation of obstacle effects on flows.

\section{References}

Branford, S., O. Coceal, T. Thomas, and S. Belcher, 2011: Dispersion of a point-source release of a passive scalar through an urban-like array for different wind directions. Bound.-Layer Meteor., 139, 367-394.

Castro, I. P., 2017: Are urban-canopy velocity profiles exponential? Bound.-Layer Meteor., 164, 337-351.

Cheng, H., P. Hayden, A. Robins, and I. Castro, 2007: Flow over cube arrays of different packing densities. J. Wind Eng. Ind. Aerodyn, 95, 715-740.

Coceal, O., and S. Belcher, 2004: A canopy model of mean winds through urban areas. Quart. J. Roy. Meteor. Soc., 130, 13491372.

Coceal, O., T. Thomas, I. Castro, and S. Belcher, 2006: Mean flow and turbulence statistics over groups of urban-like cubical obstacles. Bound.-Layer Meteor., 121, 491-519.

Coceal, O., T. G. Thomas, and S. E. Belcher, 2007: Spatial variability of flow statistics within regular building arrays. Bound.-Layer Meteor., 125, 537-552.

Goldstein, D., R. Handler, and L. Sirovich, 1993: Modeling a noslip flow boundary with an external force field. J. Comput. Phys., 105, 354-366.

Kondo, H., A. Inagaki, and M. Kanda, 2015: A new parametrization of mixing length in an urban canopy derived from a large-eddy simulation database for tokyo. Bound.-Layer Meteor., 156, 131-144.

Kono, T., T. Tamura, and Y. Ashie, 2010: Numerical investigations of mean winds within canopies of regularly arrayed cubical buildings under neutral stability conditions. Bound.-Layer Meteor., 134, 131.

Martilli, A., A. Clappier, and M. W. Rotach, 2002: An urban surface exchange parameterisation for mesoscale models. Bound.-Layer Meteor, 104, 261-304.

Martilli, A., and J. L. Santiago, 2007: CFD simulation of airflow over a regular array of cubes. Part II: Analysis of spatial average properties. Bound.-Layer Meteor., 122, 635-654.

Nakayama, H., T. Takemi, and H. Nagai, 2011: LES analysis of the aerodynamic surface properties for turbulent flows over building arrays with various geometries. J. Appl. Meteor. Climatol., 50, 1692-1712.

Rasheed, A., and D. Robinson, 2013: Characterization of dispersive fluxes in mesoscale models using LES of flow over an array of cubes. Int. J. Atmos. Sci., 2013, 1-10.

Raupach, M., and R. Shaw, 1982: Averaging procedures for flow within vegetation canopies. Bound.-Layer Meteor, 22, 7990.

Santiago, J., and A. Martilli, 2010: A dynamic urban canopy parameterization for mesoscale models based on computational fluid dynamics reynolds-averaged Navier-Stokes microscale simulations. Bound.-Layer Meteor., 137, 417439.

Xie, Z.-T., O. Coceal, and I. P. Castro, 2008: Large-eddy simulation of flows over random urban-like obstacles. Bound.Layer Meteor., 129, 1-23.

Yoshida, T., T. Takemi, and M. Horiguchi, 2018: Large-eddysimulation study of the effects of building-height variability on turbulent flows over an actual urban area. Bound.-Layer Meteor., 168, 127-153.

Manuscript received 4 August 2018, accepted 17 October 2018 SOLA: https://www.jstage.jst.go.jp/browse/solal 